\title{
Comments on the limits and internal structure of the mammalian midbrain
}

\author{
Luis Puelles \\ Department of Human Anatomy, Faculty of Medicine, University of Murcia, Murcia, Spain
}

\begin{abstract}
The anatomic concept of the midbrain has changed considerably in recent times, due to advances in molecular developmental neurobiology which have shown the mechanisms that pattern the midbrain relative to diencephalon and hindbrain, as well as relative to its inner structure. The midbrain unitary developmental field that was discovered in this way is smaller than the classic concept of the midbrain, which therefore necessarily included portions of the caudal diencephalon and the rostral prepontine hindbrain. These developmental mechanisms are summarized, the correct boundaries defined, and the diencephalic and hindbrain structures that were wrongly ascribed to the midbrain are illustrated schematically and classified properly as to their origins. Finally, the internal structure of the modern midbrain is treated systematically in terms of dorsoventral and anteroposterior regionalization.
\end{abstract}

Keywords: boundaries; derivatives; diencephalon; hindbrain; midbrain; neuromeres; patterning; regionalization

Anatomy 2016;10(1):60-70 @2016 Turkish Society of Anatomy and Clinical Anatomy (TSACA)

\section{Introduction}

Roughly 45 years ago, when I started teaching neuroanatomy, it was customary to present the brainstem as built by three blocks, the medulla oblongata, the pons (with the cerebellum) and the midbrain. The ponto-mesencephalic or caudal midbrain limit was traced along the sulcus that runs above the pons and its continuation, the middle cerebellar peduncle. The boundary passed below the lemniscal trigone (which contains the superior cerebellar peduncle and the lateral lemniscus tracts), and finished dorsally at the superior medullary velum, caudal to the trochlear nerve root. The mes-diencephalic border represented the rostral midbrain limit, which was given by an imaginary plane passing in front of the superior colliculus (behind, across, or in front of the posterior commissure, depending on the authors). This limit passed behind the medial geniculate body and ended ventrally at the upper end of the interventricular fossa, close to the mamillary bodies. The whole interpeduncular fossa and visible peduncles were held to be mesencephalic, and so were the oculomotor and trochlear nerves (Figure 1a).

This classic concept of the midbrain limits has not stood the test of time, due to accrued developmental knowledge and recent gene expression patterns implying strongly that both limits defined above are inexact. It has been shown that the old 'midbrain' does not represent a developmental unit, because it is too inclusive: it encompasses diencephalic derivatives rostrally and hindbrain derivatives caudally. A new, more restricted concept of the midbrain has emerged (Figure 1b), which is consistent with gene patterns, patterning mechanisms, and modern notions about neuromeric structure of the neural tube (the prosomeric model). This essay aims to summarize the new data and explain the resulting changes in the midbrain concept, ending with a summary of present knowledge about its inner structure (internal transversal, longitudinal and radial parts, and corresponding derivatives).

\section{Molecular Patterning of the Midbrain}

It seems convenient to summarize at the start the present embryologic knowledge about patterning of the midbrain, since this provides a causal background that necessarily applies to any morphologic considerations. Incipient differential specification of the neural ectoderm occurs along the dorsoventral and anteroposterior dimensions, starting at early neural plate stages. 
Dorsoventral patterning proceeds in general in a similar way throughout the length of the neural primordium, including naturally the midbrain (Figure 2). Antagonistic ventralizing and dorsalizing morphogen signals originated respectively at the immature floor and roof plates spread in opposed gradients across the prospective lateral wall of the neural tube, changing the specification state of the neuroepithelial cells proportionately to the relative local concentration of both signals. The principal floor plate signal is the diffusible protein sonic hedgebog $(\mathrm{SHH})$; this is first secreted by the axial mesoderm (the notochord) and induces at high concentration the differentiation of the floor plate in the overlying ectoderm; the floor plate in its turn activates likewise the $S b h$ gene, and starts to produce and release $\mathrm{SHH}$, which diffuses dorsalward along the neural wall, where it is needed in some quantity for the differentiation of the basal plate. At midbrain levels (similarly as in the whole forebrain, but not in the hindbrain), the basal plate also activates the $S h h$ gene and becomes itself a third source of $\mathrm{SHH}$. Thus the floor and basal longitudinal domains first obey and then co-produce the ventralizing $\mathrm{SHH}$ signal at the high end of its concentration gradient and minimum levels of dorsalizing signals. Subtler $\mathrm{SHH}$ effects are obtained on the alar plate, in combination with stronger dorsalizing effects generated from the roof plate. The dorsalizing morphogens spreading ventrally in a decreasing concentration gradient from the roof plate (differentiated itself at the highest concentration of these morphogens, activated by signals from the surrounding non-neural ectoderm) include members of the Wnt protein family (e.g., WNT3, 3a, 8), which antagonize directly or indirectly $\mathrm{SHH}$ signaling functions. Other genetic determinants downstream of SHH and the WNTs get secondarily involved too, adding to the generation of multiple differential dorsoventral molecular identities in the neural tube wall. Once an equilibrium of such dynamic neuroepithelial molecular states is achieved, we have in the first place a floor plate, a basal plate, an alar plate and a roof plate (the so-called primary longitudinal zones; accordingly, the significant longitudinal alar-basal boundary results fixed in position). Moreover, there are solid data suggesting that subtle details of antagonistic dorsoventral patterning cause all these primary zones to become further subdivided into two or more (up to 6) thin microzonal bands, each subtly different from the adjacent ones in molecular phenotype and histogenetic fate. The pattern of microzones can vary somewhat from one brain region to the next (due in part to differential anteroposterior patterning; see below). The midbrain thus finally has its own characteristic microzonal subdivision pattern of the 4 primary lon-
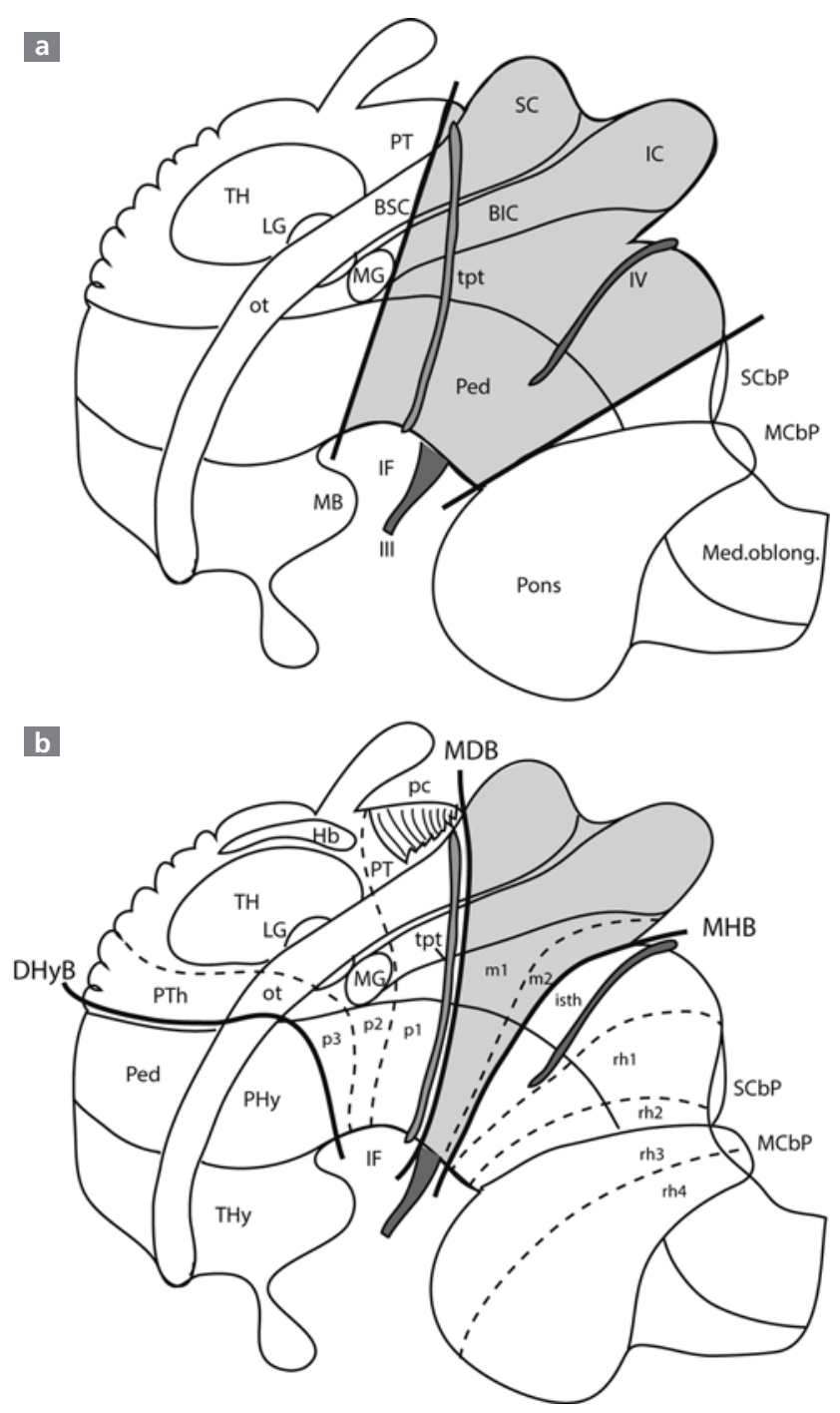

Figure 1. Diagrams illustrating the 'old' and 'new' definitions of the midbrain (color-shaded). The anatomic landmarks represented are identical in the two drawings, for comparison. The postulated boundaries with the diencephalon and the hindbrain are marked with thick black lines. The (a) version ascribes the whole interpeduncular fossa (IF) to the ventral midbrain, as well as all the prepontine portion of the brainstem. Note the apparently arbitrary position of the transverse peduncular tract, or of the oculomotor root. The $(\mathbf{b})$ version postulates a much reduced, wedgeshaped midbrain, subdivided into unequal $\mathrm{m} 1$ and $\mathrm{m} 2$ mesomeres. The mes-diencephalic boundary (MDB) and midbrain-hindbrain boundary (MHB) are marked by thick lines. In addition, the diencephalo-hypothalamic boundary is likewise indicated (DHyB), to illustrate the concept of hypothalamus conceived as a region placed rostral to the diencephalon (rather than ventral, as in a; note the transverse subdivision into peduncular and terminal hypothalamic moieties -Phy, THy). The ventral diencephalon proper participates in the rostral interpeduncular fossa, rostrally to the oculomotor roots. The diencephalon appears subdivided into three prosomeres, p1-p3, which contain dorsally the pretectum (PT), the thalamus and habenula (Th, $\mathrm{Hb}$ ) and the prethalamus (PTh), respectively. The prepontine brainstem is ascribed now to the hindbrain, being subdivided into isthmic and rh1-rh2 rhombomeres (isthmus and rh1 extend also into the cerebellum, here eliminated by section of the cerebellar peduncles). Note the trochlear nerve now turns out to be isthmic. 
gitudinal zones. In general, this characteristic midbrain dorsoventral pattern is more comparable to patterns obtaining more rostrally in the diencephalon and hypothalamus than to the hindbrain pattern. This has led to the recent suggestion to include the midbrain in the forebrain, ${ }^{[1]}$ since molecularly it is quite distinct from the hindbrain in many respects.

Anteroposterior patterning of the midbrain occurs in two steps. In the first place, the site of the prospective transverse midbrain-hindbrain boundary is established molecularly. This occurs by antagonistic interaction between a rostral domain expressing the early neural marker Otx2 and a caudal domain expressing Gbx2. At early neural plate stages the mutual boundary of these markers lies far caudally, at prospective spinal cord levels (Figure 3a). However, Gbx2-expressing caudal tissue is able to downregulate Otx2 expression and to induce its own expression in the rostrally adjacent neural tissue, thus causing rapid displacement of the Otx2/Gbx2 molecular boundary rostralward. Eventually, at late neural plate stages, this interaction reaches an equilibrium state, and the boundary between $O t \times 2$ and $G b x 2$ expression domains becomes completely fixed, precisely at the developing midbrain-hindbrain limit (Figures $\mathbf{3 b}$, $\mathbf{c}$ and $\mathbf{e}$ ). This is held to remain stable thereafter, even in the adult stage. It represents the earliest anteroposterior limit formed in the brain, and it is present even before full closure of the neural tube occurs (Figure 3b). Accordingly, a paramount reason for distinguishing anatomically the midbrain from the hindbrain is the fact that the midbrain, similarly as the rest of the forebrain, permanently expresses $O t x 2$, whereas the hindbrain does not (a minor exception is seen in Figure 3e, where a basal cell population contributing to the interpeduncular nucleus appears positive, as well as the choroidal tela of the hindbrain). The hindbrain widely expresses instead $G b \times 2$, which is absent in the midbrain. A similar mechanism protagonized by different antagonistic genes ulteriorly establishes the midbrain-diencephalon boundary (Pax6 rostrally in the diencephalon opposed to $W n t 1$ and Pax2 in the midbrain). The Pax6 marker (not shown) is also permanent, and allows to visualize from early neural tube stages onwards the prospective mes-diencephalic border.

In a second step, the isthmic secondary organizer provides anteroposterior gradiental positional information that leads competent midbrain tissue to select differential specific molecular identities; this generates anteroposterior structural differentiation within the midbrain. The isthmic organizer ( $\mathrm{IsO}_{\mathrm{s}}$ ) is a differentiated transverse band of neural tube neuroepithelium that releases into the medium fibroblast growth factor 8 (FGF8), a dif-

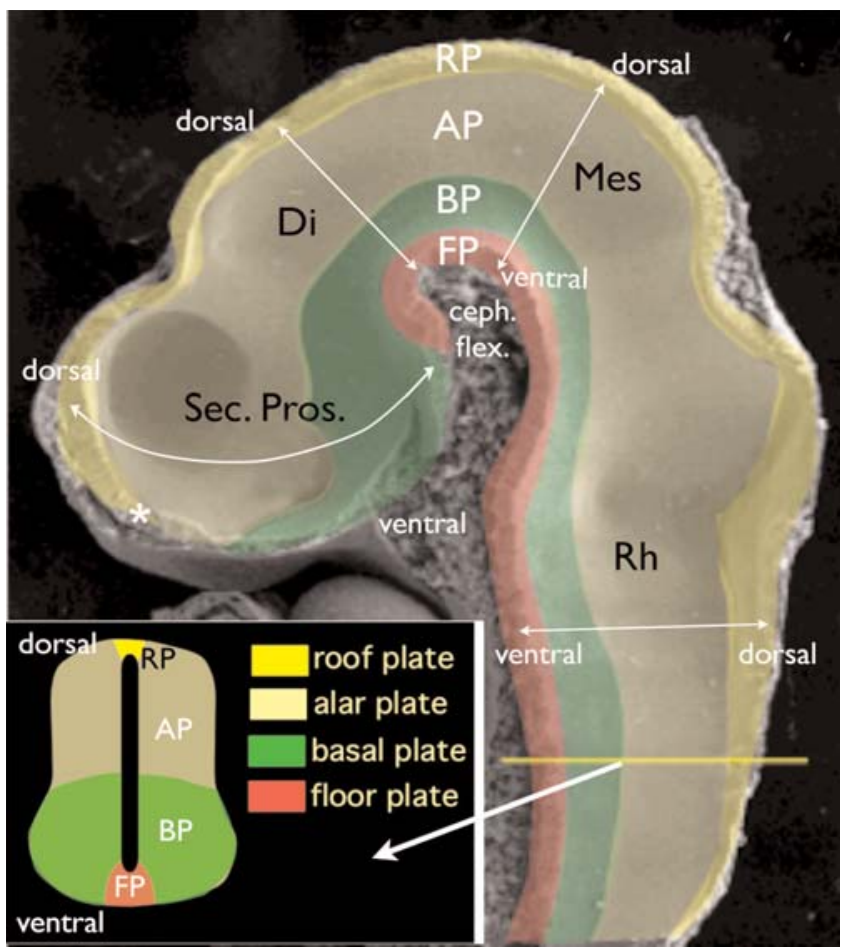

Figure 2. This drawing extracted from Martínez et al., $2012^{[28]}$ illustrates the common dorsoventral patterning process of the neural tube. Antagonistic signals spreading in opposed gradients from the roof and the floor sites lead to the formation of the primary longitudinal zones shared by all brain parts (floor, basal, alar and roof plates; see details in the text). Note the dorsoventral dimension apparently changes its direction in space in different parts of the brain, due to axial bending of the neural tube at the cephalic flexure, where the ventral ends converge (arrows). Used with kind permission. [Color figure can be viewed in the online issue, which is available at www.anatomy.org.tr]

fusible morphogen (Figure 3d). The $F g f 8$ gene is activated first at the $O t \times 2 / G b \times 2$ boundary and participates in a retroactively stabilizing signaling network with $W n t 1$ expressed in the midbrain, local Pax genes, and En1/En2 genes expressed both in the midbrain and rostral hindbrain. The functioning of this network expands initially the expression of $F g f 8$ to the whole transverse isthmus territory (excepting the floor plate) and eliminates $F g f 8$ expression within the midbrain (additional molecules are also involved in this process). Midbrain $W n t 1$ expression is initially widespread, reaching the mes-diencephalic boundary, but results substantially reduced subsequently, becoming restricted to the midbrain roof plate and a thin transverse ring just in front of the isthmo-mesencephalic boundary. WNT1 diffuses seemingly only at short-range, and an important function is to maintain midbrain neuroepithelial proliferation close to where its signal is strongest (the caudal midbrain and the roof plate). Another early WNT1 function may participate in 

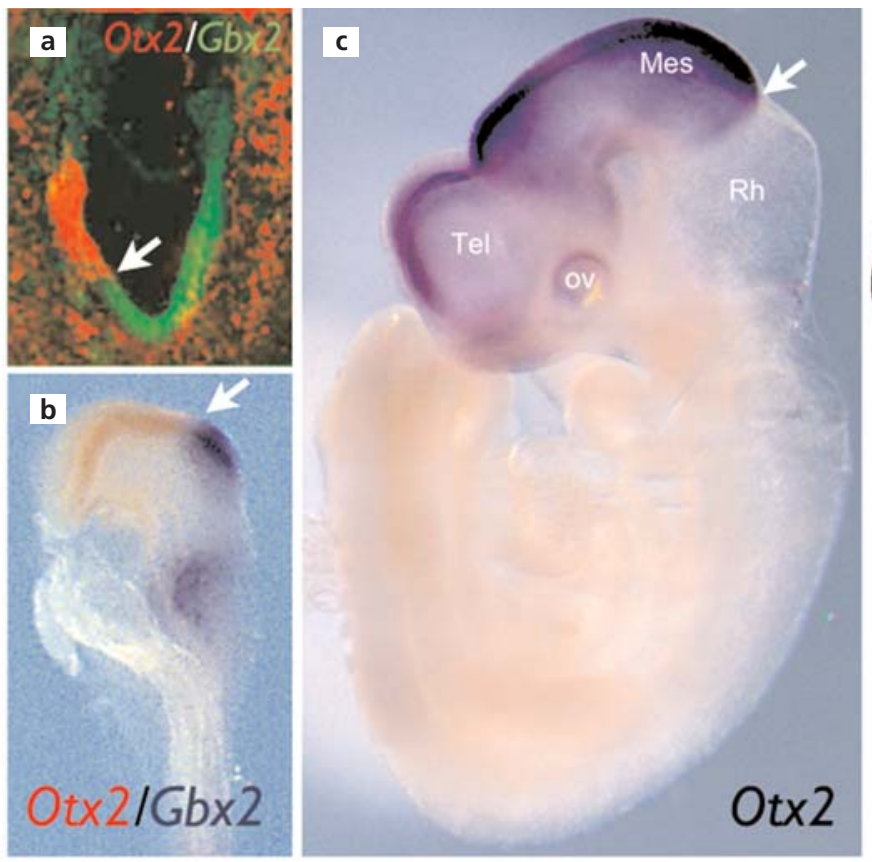

\section{E10.5}
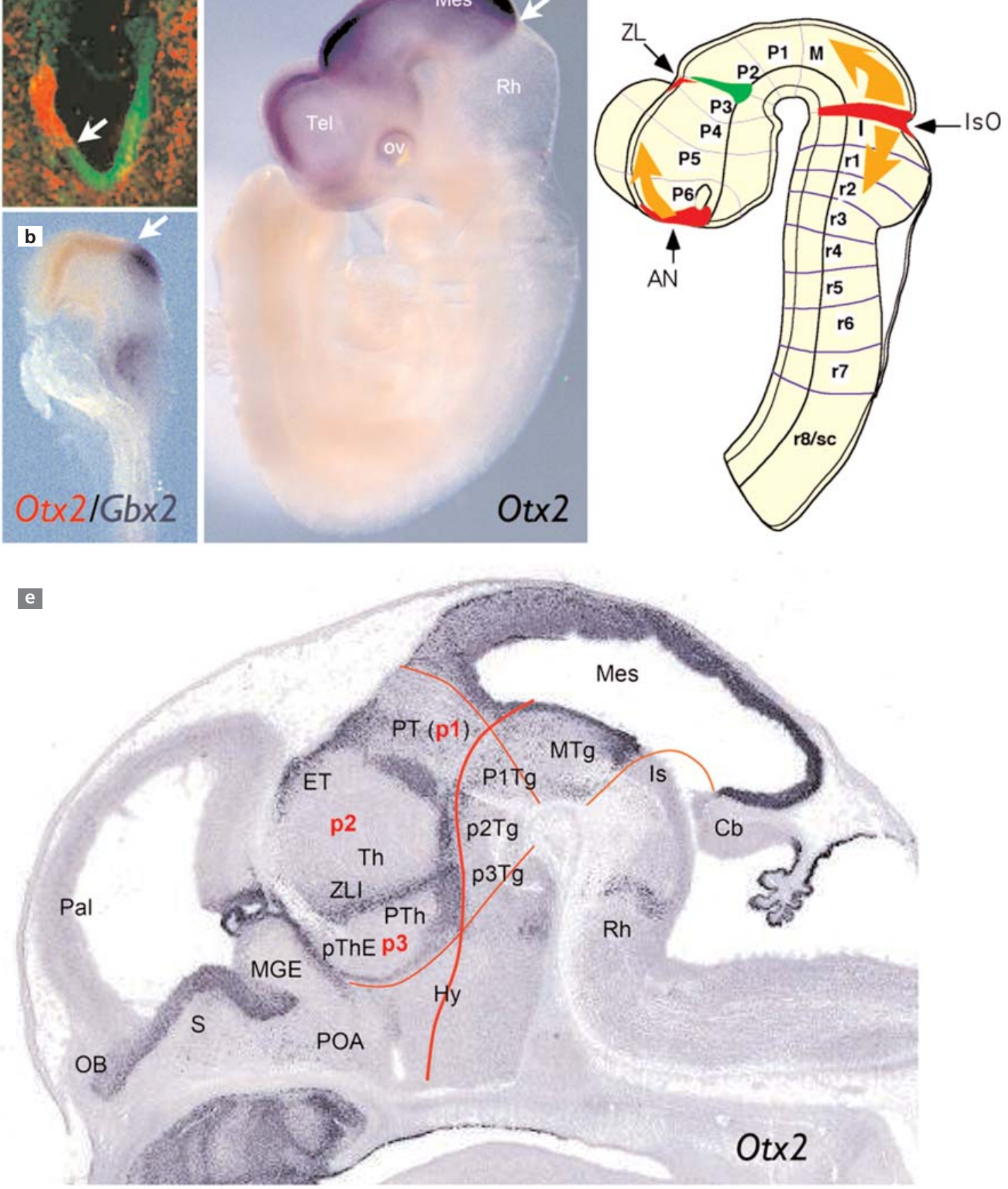

Figure 3. Pannel illustrating anteroposterior patterning of the midbrain (also extracted from Martínez et al., 2012 ${ }^{[28]}$ ). (a) and (b) show mouse embryos at early and late neural plate stages, showing color-coded expression of the Otx2 gene rostrally and the Gbx2 gene caudally. This molecular boundary moves progressively rostralward (due to antagonistic interactions between the apposed domains) until it becomes stabilized shortly before neural tube closure. (c) shows an early neural tube with Otx2 signal restricted to the forebrain, which includes the midbrain vesicle caudally. Note the sharp transverse boundary with the hindbrain (white arrow). (d) is a schema that illustrates the second phase of midbrain anteroposterior patterning that results from the emergence of Fgf8 expression selectively at the previously established Otx2/Gbx2 boundary. FGF8 protein diffuses rostralward and caudalward (large arrows), influencing the fate of both the midbrain and the prepontine hindbrain. The gradient of FGF8 obtained across the midbrain serves to produce the structural subdivisions shown in Figure $\mathbf{4 e}$ is a section through an (e) 14.5 mouse embryo, showing again Otx2 expression, which persists in time thereafter. Note the clearcut midbrain-hindbrain molecular boundary, clearly distant from the prospective pons. The Otx2 gene expression pattern also underlines the diencephalic $\mathrm{p} 1-\mathrm{p} 3$ prosomeres converging ventrally into the interpeduncular fossa (boundaries as transverse red lines), the axially bent alar-basal boundary (longitudinal red line) and the forebrain and hindbrain choroidal telae. Used with kind permission. [Color figure can be viewed in the online issue, which is available at www.anatomy.org.tr] 
antagonism with diencephalic signals in the establishment of the mes-diencephalic boundary. FGF8 molecules diffusing rostralward across the midbrain in a decreasing concentration gradient provide positional cues for the midbrain progenitor cells, enabling them to select alternative sets of differentiation genes in function of their distance from the isthmic organizer (and the local concentration of FGF8). This generates a clearcut anteroposterior series of differential structures observed in the mature alar midbrain (the superior and inferior colliculi are the largest and better known ones, but there is a total of 4 distinct domains; see below; see also the midbrain histogenetic rostrocaudal gradient shown in
Figure 3e); the position of the alar plate close to roofderived WNT1 signals causes alar territory to grow much more than the underlying basal plate, allowing also more room for reading out distinct fates along the caudorostral gradient of FGF8 (Figure 3e). This differential alar versus basal growth generates the wedge-shape of the midbrain as a whole at the apex of the cephalic flexure (Figure 3e).

We accordingly have now sufficient mechanistic understanding of how the midbrain territory emerges during early development as a developmental unit that is distinguishable molecularly and causally from both the hind-

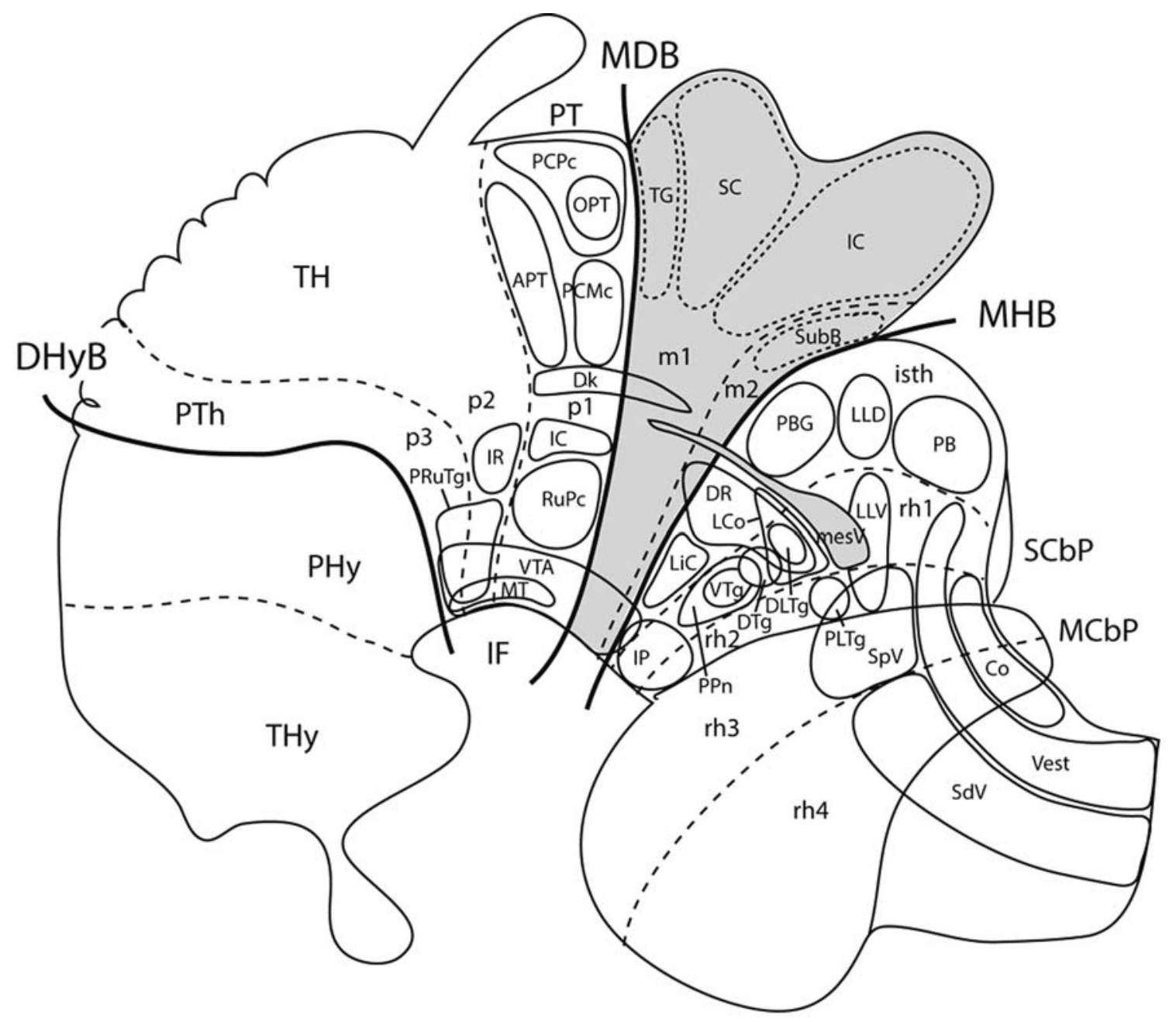

Figure 4. Drawing modelled after Figure $\mathbf{1 b}$, emphasizing the variety and relative position of grisea that used to be ascribed to the midbrain according to the 'old' concept illustrated in Figure 1a, but are now thought to belong either to the hindbrain or to the diencephalon. The prosomeric model serves to classify such grisea precisely into specific neuromeric domains. Note the mes $V$ population is interpreted here as a migrated midbrainderived structure, and therefore is color-coded as the midbrain. The later shows the relative position of the major alar centres of the $\mathrm{m} 1 \mathrm{mes}$ mere, the tectal gray (TG), the superior colliculus (SC), and the inferior colliculus (IC), as well as the major superficial derivative of the $\mathrm{m} 2 \mathrm{mesomere}$, the subbrachial nucleus (SubB; compare Figures $\mathbf{4}$ and $\mathbf{5 b}$; the latter show a schematic coronal section passing through IC, SubB and PBG in this figure, somewhat obliquely to the MHB). 
brain and the diencephalon. Moreover, we have an experimentally based theory about secondary patterning mechanisms and growth regulation telling us how the midbrain becomes regionalized into distinct neurogenetic domains along its dorsoventral and anteroposterior spatial dimensions (Figure 4), independently from neighboring hindbrain or diencephalic domains (compare Figure 1b). This knowledge leads us to the conclusion that the old midbrain concept with borders reaching significantly into the neighboring hindbrain and diencephalon territories is no longer consistent with available data and needs a conceptual readjustment (Figures 1a and $\mathbf{b}$ ).

\section{Intrusion of the Classic Midbrain into Rostral Hindbrain}

The oldest notion implying that the midbrain does not contact the upper border of the pons appeared in landmark contributions to human neuroembryology by Wilhelm His. ${ }^{[2,3]}$ He defined the isthmus territory as a transverse hindbrain region lying rostral to the pons and directly caudal to the midbrain proper. This author did not present explicitly the isthmus as a hindbrain neuromere, though his illustrations do suggest this interpretation (earlier authors had already observed multiple bulges in the neural tube wall, but the histologic concept of brain segments or neuromeres was first developed by Orr $1887,,^{[4]}$ and further explored by McClure $1890,{ }^{[5]}$ Locy $1895,{ }^{[6]}$ von Kupffer $1906,{ }^{[7]}$ Ziehen $1906,{ }^{[8]}$ and others). Palmgren $1921^{[9]}$ eventually used the neuromeric approach to perform a comprehensive developmental analysis of the midbrain in anamniote and amniote vertebrates. He concluded that the midbrain comprises two neuromeres, identified in rostro-caudal order as mesomeres 1 and 2 (m1, m2; see Figure 1b), and showed abundant evidence consistent with placing the caudal midbrain boundary in contact with the isthmus, that is, between $\mathrm{m} 2$ and the isthmus (as indicated by His $1893^{[2]}$ ). This $\mathrm{m} 2$ /isth limit passes through the narrow space that separates the oculomotor from the trochlear motor nuclei. This study thus established that the oculomotor nerve belongs to the midbrain (actually to $\mathrm{m} 1$ ), whereas the trochlear nerve must be ascribed to the isthmic hindbrain (Figure 1b). Another important conclusion was that the narrower caudal part of the interpeduncular fossa found caudal to the oculomotor nerve roots, which encloses the interpeduncular nucleus, belongs to the prepontine hindbrain, whereas the wider part of the fossa located rostral to the oculomotor roots corresponds to the diencephalic floor. This fully agrees with present-day formulations and molecular mappings offered by the prosomeric model (Figures $\mathbf{1 b}, \mathbf{3 d}$ and $\mathbf{e}, \mathbf{4}$ ), and has been corroborated by distinct gene patterns labeling the indicated interneuromeric boundaries of $\mathrm{m} 1, \mathrm{~m} 2$ and isthmus (see Puelles et al., 2012 $2^{[10]}$ ).

Other works exploring hindbrain neuromeres (or rhombomeres; abbreviated 'rh') verified Palmgren's caudal delimitation of the midbrain (e.g. Vaage 1969, 1973; Puelles and Martínez-de-la-Torre 1987; HidalgoSánchez et al., 2005). ${ }^{[11-14]}$ It was eventually concluded that the mammalian pontine nuclei develop within rh3 and rh4 (Puelles 2013; $;^{[1]}$ see Figures 1b, 4). Accordingly, it can be deduced that there exists an ample prepontine region of the hindbrain, represented by the isthmus (also referred to as rh0), plus the rh1 and rh2 neuromeric domains (Figures 1b, 4).

The whole prepontine hindbrain region (isth, rh1, rh2), excepting the cerebellum, wrongly figured as a caudal part of the midbrain in the classic concept of this brain region (Figure 1a). In addition to the trochlear motor nucleus, which we now know lies in the isthmus, other well-known brainstem cell masses were erroneously ascribed to the midbrain (Figure 4). These include the interpeduncular nuclear complex (isth, rh1), the serotonergic dorsal, caudal linear, and central raphe nuclei (isth, rh1), the parabigeminal nucleus (isth), the dorsal and ventral nuclei of the lateral lemniscus (isth and rh, respectively), the cholinergic pedunculopontine nucleus (rh1), the dorsal, ventral and dorsolateral tegmental nuclei (rh1), the parabrachial complex (isth), and the nor-adrenergic locus coeruleus (rh1), plus correlative rostral parts of the hindbrain reticular formation (Figure 4). Moreover, in mammals the major part of the so-called mesencephalic trigeminal nucleus (mesV) actually lies at the isth and rh1 regions (fewer such cells appear in the midbrain proper, particularly caudally; however, given that the homologous mes $V$ population is selectively restricted to the midbrain in reptiles and birds, it may be conjectured that the caudally displaced position of mes $\mathrm{V}$ observed in mammals may be due to embryonic tangential translocation of the corresponding cell bodies along the mes $V$ tract, in which case we would deal here with a true mesencephalic cell population displaced into the hindbrain).

On the other hand, rh2 contains the rostralmost parts of the cochlear, vestibular and trigeminal sensory columns, as well as the noradrenergic subcoeruleus nucleus, the posterodorsal tegmental nucleus, nucleus incertus, some prepontine raphe nuclear populations and corresponding reticular cell groups (Figure 4).

\section{Intrusion of the Classic Midbrain into Caudal Diencephalon}

Palmgren $1921^{[9]}$ similarly established on solid comparative developmental grounds the rostral boundary of the midbrain (the mes-diencephalic border). This corre- 
sponds to the interneuromeric boundary between the caudalmost diencephalic neuromere, called by him 'synencephalon' with mesomere 1 , as was subsequently confirmed by Rendahl 1924, ${ }^{[15]}$ who studied the diencephalic neuromeres in detail. The synencephalon is nowadays called prosomere 1 (see $\mathrm{p} 1$ in Figures $\mathbf{1 b}, \mathbf{3 d}$ and e, 4; see also Puelles $\left.2013^{[1]}\right)$. This boundary definition was corroborated as well by Vaage $1969^{[11]}$ and Puelles et al., 1987, ${ }^{[16]}$ until the fate-mapping experiments of García-López et al., 2004 ${ }^{[17]}$ and genoarchitectonic evidence reported by Ferran et al., 2007; ${ }^{[18]} 2008 ;{ }^{[19]}$ $2009^{[20]}$ helped to establish it definitively.

This boundary starts dorsally behind the posterior commissure and ends ventrally passing rostrally to the dorsal and ventral tegmental decussations and the oculomotor nerve root (Figures $\mathbf{1 b}, \mathbf{3 e}, \mathbf{4})$. The transverse peduncular tract (tpt; Figure 1b), visible in adult mammals at the surface of the cerebral peduncle, courses precisely in front of the mes-diencephalic border and is therefore diencephalic (it projects fibers of the basal optic root into the optokinetic terminal nuclei of this root, which are pretectal formations). The diencephalic synencephalon, or p1, contains in its alar plate the pretectum (PT), including the posterior commissure (pc) that crosses its roof plate over the subcommissural organ (Figures 1b, 3e, 4). The classic midbrain concept had arbitrarily included a part of the pretectum, or sometimes the whole pretectal region, in the midbrain (Figure 1a). As we noted above, a persistent Pax6 gene signal (or PAX6 immunoreaction) in the commissural pretectum certifies its diencephalic character, since the midbrain embryonic environment antagonizes that fate (there would be no pretectal formations if they accidentally came to be born inside the midbrain, under the range of the signals spreading from the isthmic organizer). The floor region of $\mathrm{p} 1$ participates in the interpeduncular fossa in front of the midbrain region, and the other two diencephalic prosomeres (p2 and p3; also characterized by distinct gene patterns) likewise locate their respective floor regions more rostrally along the interpeduncular fossa (Figures 1b, 3e, 4). This implies that the classic midbrain concept (Figure 1a) wrongly ascribed to the midbrain the whole diencephalic floor region and adjacent parts of the diencephalic basal plate. In the classic view, which was strongly influenced by the columnar model of the forebrain (Herrick 1910; ${ }^{[2]]}$ Kuhlenbeck $1973^{[22]}$ ), the diencephalic floor was thought to correspond instead to the hypothalamus (Figure 1a). This model is clearly inconsistent with present-day neuromeric, genetic and causal analysis of the forebrain in terms of dorsoventral and anteroposterior patterning (Figures 2 and 3), and therefore is considered to be obsolete (a future essay is planned to explain this complex issue).
A number of known neuronal cell groups that were classically ascribed to the midbrain belong instead to the diencephalon (particularly to the basal and floor parts of the diencephalon, but also to the alar pretectum; Figure 4). A peculiar case is represented by the substantia nigra compacta and the ventral tegmental area, classically held to be strictly mesencephalic formations containing dopaminergic neurons (SNC, VTA). They are accompanied throughout their length by a superficial population of GABAergic cells known as substantia nigra reticulata (SNR), also classically held to be strictly mesencephalic. In contrast, modern neuromeric and genoarchitectonic analysis has shown conclusively that this complex is plurineuromeric (i.e., extends across a series of neuromeres). Both VTA and SNC stretch rostralward from the isthmus (rh0), which contains the caudalmost parts, across midbrain $\mathrm{m} 2$ and $\mathrm{m} 1$, and continue into diencephalic p1-p3 (see VTA in Figure 4; review in Puelles $\left.2013^{[1]}\right)$. The diencephalic and isthmic portions of VTA and SNC/SNR were thus classically wrongly ascribed to the midbrain. Interestingly, the $\mathrm{m} 1$ and $\mathrm{m} 2$ portions of the complex, lying at rubral and retrorubral levels, respectively, are morphologically distinct (Puelles et al., $2012 ;{ }^{[10]}$ see below under Structure). The 'posterior bypothalamus' and/or 'prerubral tegmentum' (PRuTg in Figure 4) mentioned in the literature are neural regions largely derived from $\mathrm{p} 2$ and $\mathrm{p} 3$ (insofar as the parvocellular red nucleus, the rostralmost rubral entity, lies in basal p1; RPc in Figure 4). I can list under this category of diencephalic centers wrongly ascribed to the midbrain (Figure 4) also the nucleus of the basal optic root (also called medial terminal nucleus; p1-p3; MT in Figure 4), the rostral interstitial nucleus ( $\mathrm{p} 2$; RI), the interstitial nucleus of Cajal (p1; IC), the rostral part of the nucleus of Darkschewitsch ( 1 1; Dk), the nuclei of the posterior commissure (magno- and parvocellular; p1; PCMc, PCPc), and any pretectal nuclei ascribed to the midbrain (p1; APT, OPT. etc.).

In connection with the wrong inclusion of pretectal formations in the classic midbrain concept, it should be explained that there is a layered retinorecipient mesencephalic formation placed rostral to the equally retinorecipient superior colliculus, which is called the 'tectal gray' in recent literature (TG in Figure 4). This used to be called in older literature the 'posterior pretectal nucleus'. That name was incorrect, insofar as the pretectal gray clearly develops behind the posterior commissure, and therefore belongs properly to the midbrain (i.e., is not a pretectal nucleus at all, though it lies in front of the superior colliculus). The confusing wrong name should not be used any longer. 


\section{Internal Structure of the Midbrain}

As regards its internal structure, the modern midbrain is understood as constituted by derivatives of mesomeres 1 and 2 (Figures 1b, $\mathbf{4}$ and $\mathbf{5}$ ). This was suggested initially by Palmgren $1921^{[9]}$, and was variously corroborated and mapped in modern times by Vaage $1969,{ }^{[1]} 1973,{ }^{[12]}$ Hidalgo-Sánchez et al., 2005, ${ }^{[14]}$ Puelles and Martínez-dela-Torre 1987, ${ }^{[13]}$ and Puelles et al., 2012. ${ }^{[10]}$ The $\mathrm{m} 2$ derived domain was called preisthmus by Hidalgo-Sánchez et al., $2005,{ }^{[14]}$ to emphasize its location relative to the hindbrain isthmus. The $\mathrm{m} 1$ segment lying rostral to $\mathrm{m} 2$ is considerably larger than the thin preisthmus, and it contains most structures that can be properly ascribed to the midbrain (Figure 5). Accordingly, fewer elements belong to $\mathrm{m} 2$ (Figures 5a and $\mathbf{b}$ ).

Roof plate: The midbrain roof plate is represented by an astroglial palisade found along the dorsal midline. It passes between the right and left collicular bulges and stretches from the back of the pretectal posterior commissure to the isthmic roof (marked by the emergence of the trochlear nerve root). It is crossed by three distinct commissural fiber packets, which originate in the adjacent alar territories: the commissure of the tectal gray (tectal gray), the tectal commissure (superior colliculus) and the intercollicular commissure (inferior colliculus; icc in Figure 5b); see Figure 10.9 in Puelles et al., 2012. ${ }^{[10]}$

Alar plate: This wide longitudinal zone appears divided dorsoventrally into dorsal, dorsolateral and ventrolateral longitudinal subzones along both $\mathrm{m} 1$ and $\mathrm{m} 2$ (Figure 5a; Puelles et al., 2012 $\left.2^{[10]}\right)$. Considering the radial dimension of the alar plate, we distinguish throughout a well-developed inner stratum, formed by the periaqueductal gray (PAG; whose dorsal, dorsolateral and ventrolateral subzones each have distinct cell subpopulations, connections and functions), and a variously layered outer stratum, which may be called the tectal plate (also showing the corresponding three dorsoventral subzones).

The PAG contains a profuse plexus of mostly unmyelinated fibers intermixed with a variety of small and medium-size neurons (sometimes forming denser aggregates). It relates functionally to the organization of instinctive behavioral reactions in social situations requiring either passivity (submissiveness, death simulation) or intense activity (fight, flight). The PAG mentioned in the literature is plurineuromeric and extends beyond the midbrain limits rostrally and caudally (there is a pretectal PAG and an isthmic PAG, apart of the midbrain $\mathrm{m} 1$ and $\mathrm{m} 2 \mathrm{PAG}$ parts, which all are subtly distinct histologically).

As regards the tectal plate, its dorsal subzone is formed by a longitudinal nuclear formation described recently as the dorsal and ventral tectal paracommissural nuclei; ${ }^{[10]}$ these were first reported as a 'tectal longitudinal column' by Saldaña et

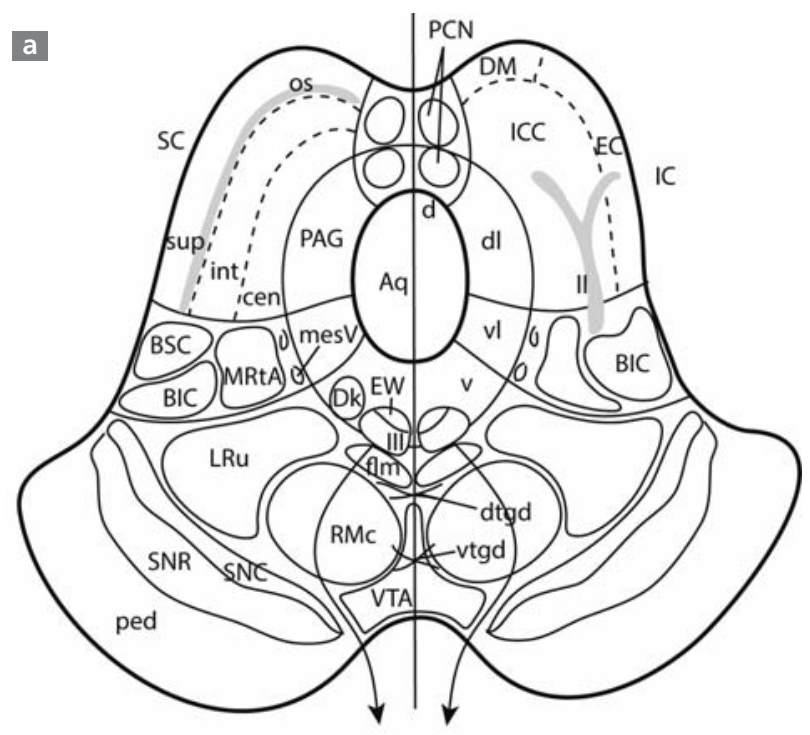

b

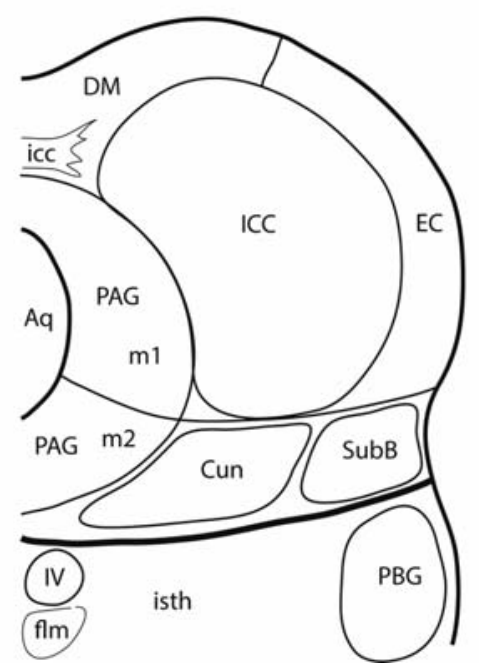

Figure 5. Schematic drawings illustrating the internal structure of the 'new' midbrain. The (a) panel displays a cross-section, whose left half intersects the superior colliculus, whereas the right half shows the inferior colliculus. Both halves correspond to the $\mathrm{m} 1$ mesomere (compare Figure 4) and are therefore similar in several aspects. The alar plate presents deeply the periaqueductal gray (PAG) and superficially the tectal plate, which contains the TG, SC and IC units shown in Figure 4, as well as some other elements. There is a dorsoventral partition into dorsal, dorsolateral and ventrolateral sectors of both strata. See further details in the text. The basal plate contains the ventral PAG with associated oculomotor complex and nucleus of Darkschewitsch (III, EW, Dk), placed over the fasciculus longitudinalis medialis ( $f(m)$ tract. More superficially there appears the magnocellular red nucleus and the laterorubral nucleus (RMc, LRu). Finally, the ventral tegmental area (VTA) occupies the subpial stratum of the floor plate next to the oculomotor nerve roots (arrows) and medial to the substantia nigra compacta et reticulata, migrated into a subpeduncular position (SNC, SNR, ped). The (b) panel illustrates a coronal section through the midbrain-isthmus transition (compare plane in Figure 4), aiming to illustrate the major derivatives of the preisthmus or alar $\mathrm{m} 2$ region. Superficially to the local PAG portion there appears the cuneiform area (Cun) and the subpial subbrachial nucleus (SubB; also illustrated in Figure 4). The parabigeminal nucleus is a similarly superficial isthmic derivative (compare also Figure 4). 
al., 2007 ${ }^{[23]}$ Figure 5a). The names we prefer refer explicitly to the position next to the tectal gray, tectal and intercollicular commissures across the tectal roof plate. The connections of these nuclei relate to the auditory pathway. ${ }^{[2]}$

The dorsolateral subzone of the tectal plate contains the major $\mathrm{m} 1$ derivatives, forming a rostrocaudal series of three large stratified entities that includes the above-mentioned tectal gray, the superior colliculus and the inferior colliculus (TG, SC, IC; Figure 4). The TG lies just behind the mes-diencephalic border, rostrally to the larger SC, and the IC limits caudally with the preisthmus ( 2 2; Figure 4). Both TG and SC are retinorecipient in their respective superficial stratum (final targets of the optic tract), but their layering schema (central, intermediate and superficial layers; see SC in Figure 5a) is slightly different; they each express some differential gene markers, and they represent in a mirror pattern the naso-temporal axis of the ordered retinal projection (nasal input is represented rostrally in TG and caudally in SC). The TG may be related functionally to the computation of continued gaze fixation upon a point in visual space, whereas the SC notoriously computes saccadic reflex conjugated eye movements to points of space of novel interest, triggered particularly by moving visual stimuli, but also by auditory and tactile cues reaching likewise the SC. Both functions can be controlled cortically. The SC has a variety of ascending (into p1-p3), local (m1), and descending (hindbrain) ipsi- and contralateral connections, and eventually contacts pre-oculomotor reticular neurons (e.g., interstitial nucleus of Cajal) rather than oculomotor motoneurons for its gaze control. The IC is organized into a rounded central nucleus and several peripheral nuclei at its superficial stratum (e.g., dorsomedial, external cortical, caudal cortical; Figures 5a and b). The IC is the target of the ascending hindbrain auditory pathway (the lateral lemniscus) and relays its output via the brachium of the IC to the SC, pretectum and thalamus (medial geniculate body; Figures 1a and $\mathbf{b}$ ); there is an interstitial bed nucleus of this ascending IC projection tract, joined rostrally by a bed nucleus of the brachium of the superior colliculus (BIC, BSC; Figures 1a and 5a). There is also a cell population receiving ascending somatosensory input from the lower brainstem and spinal cord that lies intercalated transversally between the SC and the IC; it is known as the intercollicular nucleus (ICo; not shown). Interactions between IC, ICo and SC mediate the reaction of the SC to other than visual stimuli.

The ventrolateral subzone of the $\mathrm{m} 1$ tectal plate is occupied by the bed nuclei of the two brachia, as well as by the alar part of the midbrain reticular formation (MRtA; Figure 5a), which is traversed by the ascending spinothalamic, trigeminothalamic, and medial lemniscus pathways on their way to the pretectum and thalamus.
The alar derivatives of the preisthmus (m2) are not well known, and often are mixed with the caudally adjacent isthmic cell masses (Figures $\mathbf{4}$ and $\mathbf{5 b}$ ). They include the preisthmic alar $P A G$ (divided as in $\mathrm{m} 1$ into dorsal, dorsolateral and ventrolateral longitudinal subzones; Figure 5b). The PAG is covered by a tectal plate which was classically named the 'cuneiform nuclear complex, area, or gray', a heterogeneous domain intercalated in conventional coronal sections between the IC and the isthmic nuclei (e.g., the parabigeminal nucleus). I think that the tectal paracommissural nuclear formation probably ends caudally in the preisthmus (dorsal subzone). The preisthmic dorsolateral zone remains so far unclear (the nucleus sagulum might occupy it, at least in part, unless it is wholly isthmic). The cuneiform gray may be ascribed tentatively to the ventrolateral preisthmic subzone. It can be divided into inner and outer cell masses, corresponding respectively to central and superficial strata. The superficial nucleus, thought to be homologous with the magnocellular preisthmic nucleus of birds and reptiles, was recently named subbracbial nucleus, to emphasize its position immediately caudoventral to the BIC in mammals (SubB; Figures $\mathbf{4}$ and $\mathbf{5 b}$; see also Puelles et al., 2012 ${ }^{[10]}$ ). The name cuneiform area would thus conveniently remain restricted to the deeper cell mass, which represents the preisthmic component of the alar midbrain reticular formation (Cun; Figure 5b).

Basal and floor plates: The basal plate derivatives of $\mathrm{m} 1$ include periventricularly the ventral subdivision of the $P A G$, which shows bilaterally a distinct rounded subarea known as the nucleus of Darkschewitsch (Dk; Figure 5a; this is its caudal part, since there is also a major rostral part in the pretectal PAG; Dk in Figure 4). It is formed exclusively by GABAergic neurons. Intercalated between the ventral PAG and the intermediate stratum of the basal plate (tegmentum), which is in general occupied by the midbrain basal reticular formation, there appears the oculomotor nuclear complex, formed by the main oculomotor nucleus, that innervates extra-ocular muscles, and the parasympathetic preganglionic accessory (Edinger-Westphal) nucleus, which innervates the ciliary ganglion (III; EW; Figure 5a). The latter controls the intra-ocular involuntary smooth muscles of the pupil (miosis) and ciliary body (accommodation). At the midline (floor plate) between the main oculomotor nuclei there occurs the decussation of the crossed component of the oculomotor nerve (targeting the eye muscles directing gaze upwards). This decussation curiously results from migration of the motoneuron cell bodies across the midline, so that the preformed axons are passively drawn trailing to the other side. ${ }^{[24-26}$ Ventrally to this level there appears the voluminous dorsal tegmental decussation, formed by crossing tectoisthmic, tectopontine, tectobulbar and tectospinal axons (Figure 5a). The rostral linear nucleus is a small reticular population associated to this 
area (not shown; note the caudal linear nucleus is isthmic; Figure 4). Superficially to the oculomotor complex there is a specialized area of the reticular formation which forms the magnocellular red nucleus (or nucleus ruber magnocellularis; RMc in Figure 5a). It receives crossed cerebellar input (nucleus interpositus) via the brachium conjunctivum ascending into the thalamus after its decussation across the isthmic floor plate, among whose fibers its cells lie interstitially; this nucleus originates the crossed rubrospinal tract passing through the local ventral tegmental decussation (Figure 5a). The midbrain magnocellular red nucleus (restricted to basal $\mathrm{m} 1$ ) needs to be distinguished from the parvocellular red nucleus found in the pretectal basal plate (RPc in Figure 4); the latter receives crossed dentate nucleus input from the brachium conjunctivum as it passes through p1, and generates the ipsilateral rubro-olivary tract; it is limited rostrally by the retroflex tract, a landmark of the $\mathrm{p} 2 / \mathrm{p} 1$ boundary. The midbrain red nucleus is surrounded laterally by the laterorubral nucle$u s$, which is a differentially specialized area of the basal midbrain reticular formation (LRu; Figure 5a). The latter limits laterally with the alar reticular formation described above under the ventrolateral alar subzone.

Superficially to the reticular intermediate stratum of the basal midbrain there appears bilaterally the substantia nigra formation (SNC, SNR), covered by the prominent peduncular tracts, also known classically as pes pedunculi (they represent cortico-spinal, cortico-nuclear and cortico-pontine fibers). Medially, across the floor plate, there appears the dopaminergic ventral tegmental area, in contact with the mesencephalic part of the interpeduncular fossa, where the oculomotor nerve roots emerge after arching through the red nucleus (Figure 5a). The dopaminergic neurons of the VTA and SNC in general are produced at a floor plate progenitor domain found singularly across the broadening interpeduncular fossa present at the isth, $\mathrm{m} 1, \mathrm{~m} 2$, and $\mathrm{p} 1-\mathrm{p} 3$ neuromeres (the brain floor plate otherwise scarcely produces neurons). I deal here strictly with the mesencephalic $\mathrm{m} 1$ and $\mathrm{m} 2$ parts (compare Figure 4). Once produced, the dopaminergic neurons migrate radially through the floor plate domain into the local superficial stratum. Some neurons mature at this location, forming the VTA, whereas others migrate tangentially and subpially into the right and left basal plate regions, where the SNC is formed (this is the so-called 'inverted fountain' migration). The lateralmost elements of the SNC possibly invade the neighboring alar plate. The equivalent $\mathrm{m} 2$ dopaminergic population is formed similarly at the $\mathrm{m} 2$ floor plate and the derivatives aggregate at the $\mathrm{m} 2$ part of the VTA, which represents the distinct interfascicular nucleus, which projects to the habenula via the retroflex tract, found at the midline between the two retroflex tracts coursing backwards into the prepontine interpeduncular nucleus (IP; Figure 4), and also migrate laterally and subpially to form a thin transversal band of radially disposed dopaminergic cells found caudal to the red nucleus and rostral to the isthmus. This is the retrorubral A8 cell group of the literature (see Figure 10.10 in Puelles et al., 2012 ${ }^{[10]}$ ). The $\mathrm{m} 1 \mathrm{SNC}$ and VTA counterparts represent instead the conventional $A 9$ and $A 10$ cell groups, respectively, which have differential forebrain targets. The GABAergic SNR cells are produced separately along the basal plate of isth, $\mathrm{m} 1, \mathrm{~m} 2$ and $\mathrm{p} 1-\mathrm{p} 3$. They migrate radially across the tangentially migrated SNC primordium, acquiring secondarily a position superficial to it (some cells remain intermixed). It has been described that these SNR elements may not remain restricted to the neuromeres where they are produced, so that isthmic elements may later appear within topographically mesencephalic or diencephalic parts of the plurineuromeric complex. There is some evidence that different neuromeric sectors of the SNR express given gene markers selectively.

The literature places the whole dorsal raphe nucleus, an important serotonergic cell midline aggregate, within the midbrain. However, detailed genoarchitectonic descriptive studies and transgenic lineage tracings have shown conclusively that the major part of this complex belongs to the isthmus and rh1 domains, as occurs with the caudal linear nucleus (review in Alonso et al., 2013 $\left.;^{[27}\right]$ see Figure 4). At early embryonic stages all serotonergic cells seem restricted to the hindbrain, where they are produced along the longitudinal band of $N k x 2.2$-expressing basal neuroepithelium lying next to the floor plate. $N k \times 2.2$ expression is induced by high SHH levels, a morphogen that exclusively diffuses out of the floor plate in the hindbrain. This band does not exist properly in the midbrain, since there (as in the rest of the forebrain) the basal plate also activates the $S h b$ gene, and produces itself large amounts of diffusing SHH. Induction of Nkx2.2 in the midbrain therefore occurs at or next to the alar-basal boundary, where the combined gene codes lead cell fates in a different direction. In principle, therefore, the midbrain should not display any serotonergic neurons, as occurs elsewhere in the forebrain. However, a small rostral prolongation of the isthmic dorsal raphe population appears to invade the $\mathrm{m} 2$ floor plate at advanced embryonic stages; this possibly implies a rostralward migration of some isthmic serotonergic cells, which eventually form a midbrain part of the dorsal raphe complex (DR; Figure 4; see Alonso et al., 2013, ${ }^{[27]}$ Puelles et al., 2012 $\left.{ }^{[10]}\right)$.

\section{Acknowledgements}

This study was supported by MICINN grant BFU201457516P (with European FDR support) and SENECA Foundation contract 19904/GERM/15. 


\section{References}

1. Puelles L. Plan of the developing vertebrate nervous system, relating embryology to the adult nervous system (Prosomere model, overview of brain organization). In: Rubenstein JLR, Rakic P, editors. Comprehensive developmental neuroscience: Patterning and cell type specification in the developing CNS and PNS. Amsterdam: Academic Press; 2013. p. 187-209.

2. His W. Über das frontale Ende des Gehirnrohrs. Archiv für Anatomie und Entwicklungsgeschichte [Anatomische Abteilung des Arch. f. Anat. u. Physiol. 1893b] 1893;3:157-71.

3. His W. Die Entwicklung des menschlichen Gehirns während der ersten Monate. Leipzig: Hirzel Verlag; 1904.

4. Orr HJ. Contribution to the embryology of the lizard; with especial reference to the central nervous system and some organs of the head; together with observations on the origin of the vertebrates. J Morphol $1887 ; 1: 311-72$.

5. Charles FW, McClure BA. The segmentation of the primitive vertebrate brain. J Morphol 1890;4:35-56.

6. Locy WA. Contribution to the structure and development of the vertebrate head. Boston: Ginn and Co; 1895.

7. von Kupffer C. Die Morphogenie des Zentralnervensystems. In: Hertwig O, editor. Handbuch der vergleichenden und experimentellen Entwicklungslehre der Wirbeltiere. Vol. 2, Part 3. Jena: Fischer Verlag; 1906. p. 1-272.

8. Ziehen T. Die Morphogenie des Zentralnervensystems der Säugetiere. In: Hertwig O, editor. Handbuch der vergleichenden und experimentellen Entwicklungslehre der Wirbeltiere. Vol. 2, Part 3. Jena: Fischer Verlag; 1906. p. 273-351.

9. Palmgren A. Embryological and morphological studies on the midbrain and cerebellum of vertebrates. Acta Zool 1921;2:1-94.

10. Puelles E, Martinez-de-la-Torre M, Watson C, Puelles L. Midbrain. In: Watson C, Paxinos G, Puelles L editors. The mouse nervous system. San Diego: Academic Press Elsevier; 2012. p. 337- 59.

11. Vaage $\mathrm{S}$. The segmentation of the primitive neural tube in chick embryos (Gallus domesticus). A morphological, histochemical and autoradiographical investigation. Ergebn Anat Entwicklungsgesch 1969;41:3-87.

12. Vaage $\mathrm{S}$. The histogenesis of the isthmic nuclei in chick embryos (Gallus domesticus). I. A morphological study. Z Anat Entwicklungsgesch 1973;142:283-314.

13. Puelles L, Martínez-de-la-Torre M. Autoradiographic and Golgi study on the early development of $\mathrm{n}$. isthmi principalis and adjacent grisea in the chick embryo: a tridimensional viewpoint. Anat Embryol (Berl) 1987; 176:19-34.

14. Hidalgo-Sánchez M, Martínez-de-la-Torre M, Alvarado-Mallart RM, Puelles L. Distinct pre-isthmic domain, defined by overlap of
Otx2 and Pax2 expression domains in the chicken caudal midbrain. J Comp Neurol 2005;483:17-29.

15. Rendahl H. Embryologische und morphologische Studien über das Zwischenhirn beim Huhn. Acta Zool 1924;5:241-344.

16. Puelles L, Amat JA, Martínez-de-la-Torre M. Segment-related, mosaic neurogenetic pattern in the forebrain and mesencephalon of early chick embryos. I. Topography of AChE-positive neuroblasts up to stage HH18. J Comp Neurol 1987;266:147-268.

17. García-Lopez R, Vieira C, Echevarria D, Martinez S. Fate map of the diencephalon and the zona limitans at the 10-somites stage in chick embryos. Dev Biol 2004;268:514-30.

18. Ferran JL, Sánchez-Arrones L, Sandoval JE, Puelles L. A model of early molecular regionalization in the chicken embryonic pretectum. J Comp Neurol 2007;505:379-403.

19. Ferran JL, Sánchez-Arrones L, Bardet SM, Sandoval J, Martínez-dela-Torre M, Puelles L. Early pretectal gene expression pattern shows a conserved anteroposterior tripartition in mouse and chicken. Brain Res Bull 2008;75:295-8.

20. Ferran JL, de Oliveira ED, Merchán P, Sandoval JE, SánchezArrones L, Martínez-De-La-Torre M, Puelles L. Geno-architectonic analysis of regional histogenesis in the chicken pretectum. J Comp Neurol 2009;517:405-51.

21. Herrick CJ. The morphology of the forebrain in amphibia and reptilia. J Comp Neurol 1910;20:413-547.

22. Kuhlenbeck H. The central nervous system of vertebrates. Vol. 3, Part II: overall morphological pattern. Basel (Switzerland): Karger; 1973.

23. Saldaña E, Viñuela A, Marshall AF, Fitzpatrick DC, Aparicio MA. The TLC: a novel auditory nucleus of the mammalian brain. J Neurosci 2007;27:13108-16.

24. Puelles L, Malagón F, Genis-Gálvez JM. The migration of oculomotor neuroblasts across the midline in the chick embryo. Exp Neurol 1975;47:459-69.

25. Puelles L, Privat J. Do oculomotor neuroblasts migrate across the midline in the fetal rat brain? Anat Embryol (Berl) 1977;150:187-206.

26. Puelles L. A Golgi-study of oculomotor neuroblasts migrating across the midline in chick embryos. Anat Embryol (Berl) 1978;152:205-15.

27. Alonso A, Merchán P, Sandoval JE, Sánchez-Arrones L, GarciaCazorla A, Artuch R, Ferrán JL, Martínez-de-la-Torre M, Puelles L. Development of the serotonergic cells in murine raphe nuclei and their relations with rhombomeric domains. Brain Struct Funct 2013;218:1229-77.

28. Martínez S, Puelles E, Puelles L, Echevarria D. Molecular regionalization of developing neural tube. In: Watson C, Paxinos G, Puelles L, editors. The mouse nervous system. San Diego: Academic Press Elsevier; 2012. p. 2-18.

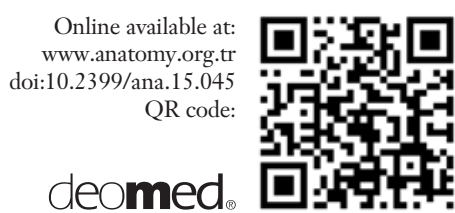

Correspondence to: Luis Puelles, MD, PhD

Department of Morphological Sciences,

Faculty of Medicine, University of Murcia,

E30100, Murcia, Spain

e-mail: puelles@fcu.um.es

Conflict of interest statement: No conflicts declared.

This is an open access article distributed under the terms of the Creative Commons Attribution-NonCommercial-NoDerivs 3.0 Unported (CC BY-NCND3.0) Licence (http://creativecommons.org/licenses/by-nc-nd/3.0/) which permits unrestricted noncommercial use, distribution, and reproduction in any medium, provided the original work is properly cited. Please cite this article as: Puelles L. Comments on the limits and internal structure of the mammalian midbrain. Anatomy 2016;10(1):60-70. 